\title{
Survey on Single image Super Resolution Techniques
}

\author{
Rujul R. Makwana ${ }^{1}$, Nita D. Mehta ${ }^{2}$ \\ ${ }^{I}(P G$ Student, EC Department, Government Engineering College, Surat, India) \\ ${ }^{2}$ (Associate Professor, EC Department, Government Engineering College, Surat, India)
}

\begin{abstract}
Super-resolution is the process of recovering a high-resolution image from multiple lowresolutionimages of the same scene. The key objective of super-resolution (SR) imaging is to reconstruct a higher-resolution image based on a set of images, acquired from the same scene and denoted as 'lowresolution' images, to overcome the limitation and/or ill-posed conditions of the image acquisition process for facilitating better content visualization and scene recognition. In this paper, we provide a comprehensive review of existing super-resolution techniques and highlight the future research challenges. This includes the formulation of an observation model and coverage of the dominant algorithm - Iterative back projection.We critique these methods and identify areas which promise performance improvements. In this paper, future directions for super-resolution algorithms are discussed. Finally results of available methods are given.

Keywords: Super-resolution, POCS, IBP, Canny Edge Detection
\end{abstract}

\section{Introduction}

Super resolution is a method for reconstructing a high resolution image from several overlapping lowresolution images[1]. The low resolution input images are the result of re-sampling a high resolution image. The goal is to find the high resolutionimage which, when re-sampled in the lattice of the input images according to the imaging model, predicts well the low resolution input images.

The success of super resolution algorithm is highly dependent on the accuracy of the model of the imaging process.If, for example, the motion computed for some of the images is not correct, the algorithm may degrade the image rather than enhance it.One solution proposed to handle local model inaccuracies and noise is regularization [2].In most cases the enforced smoothness results in the suppression of high-frequency information, and the results are blurred. Regularization may be successful when the scene is strongly restricted, e.g. a binary text image[2].

Applications for super-resolution abound. NASA has been using super-resolution techniques for years to obtain more detailed images of planets and other celestial objects. Closer to home, super-resolution can be used to enhance surveillance videos to more accurately identify objects in the scene. One particular example of this are systems capable of automatically reading license plate numbers from severely pixelated video streams. Another application is the conversion of standard NTSC television recordings to the newer HDTV format which is of a higher resolution.

A variety of approaches for solving the super-resolution problem have been proposed. Initial attempts worked in the frequency domain, typically recovering higher frequency components by taking advantage of the shifting and aliasing properties of the Fourier transform. Deterministic regularization approaches, which work in the spatial domain, enable easier inclusion of $a$ priori constraints on the solution space (typically with a smoothness prior). Stochastic methods have received the most attention lately as they generalize the deterministic regularization approaches and enable more natural inclusion of prior knowledge. Other approaches include nonuniform interpolation, projection onto convex sets, iterative back projection, and adaptive filtering. With the increased emphasis on stochastic techniques has also come increased emphasis on learning priors from from example data rather than relying on more heuristicallyderived information.

The paper is organized as follows. Section 2 presents Super Resolution Processing and a description of the general model of imaging systems (observation model) that provides the SR image formulations. Section 3 presents the SR image reconstruction approaches that reconstruct a single high-resolution image from a set of given low-resolution images acquired from the same scene. Section 4 presents Results and Discussion. Section 5 discusses several research challenges that remain open in this area for future investigation. Finally, Section.6 concludes this paper.

\section{Super-Resolution Processing}

Given a set of low resolution images that result from the observation of the same scene from slightly different views, super resolution algorithm produce a single high resolution image by fusing the input LR images such that the final HR image reproduces the scene with a better fidelity than any of the LR images[11]. 
The central idea in super resolution processing is to convert the temporal resolution in to spatial resolution. In broad sense, this approach can be used to perform any combination of the following image processing tasks:

- Registration

- Interpolation

- De-bluriring

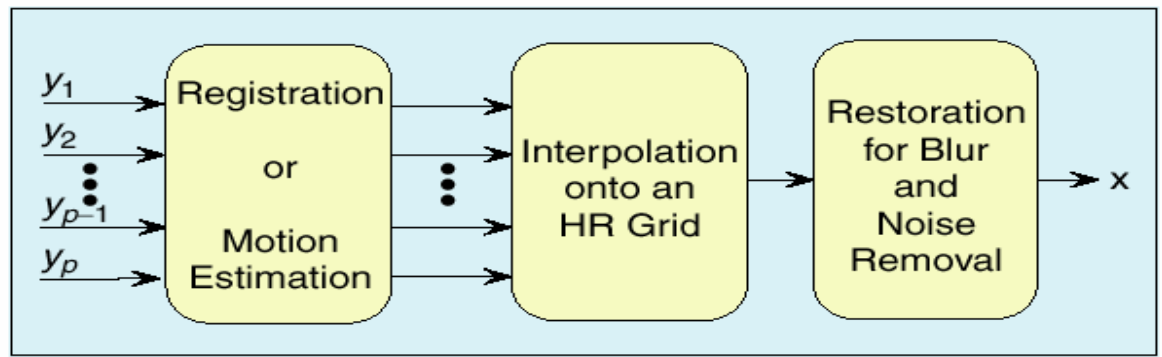

Fig.1 Phases of Super-Resolution[3]

First, the SRR algorithm receive several low-resolution corrupted images as the inputs then the registration or Motion Estimation process estimate the relative shifts between LR images compared to the reference LR image with fractional pixel accuracy. Obviously, accurate sub-pixel motion estimation is a very important factor in the success of the SRR algorithm. Since the shifts between LR images are arbitrary, the registered HR image will not always match up to a uniformly spaced HR grid. Thus, non-uniform interpolation is necessary to obtain a uniformly spaced HR image from a composite of non-uniformly spaced LR images. Finally, image restoration(De-blurring) is applied to the up-sampled image to remove blurring and noise.Before presenting the review of existing SR algorithms, we first model the LR image acquisition process.

\subsection{Observation Model}

Based on the most common observation model (Fig.2) it is considered that the available low-resolution input images are obtained from the high-resolution original scene by warping, blurring and down sampling the scene. Consider the desired HR image of size $L_{1} N_{1} \times L_{2} N_{2}$ written in lexicographical notation as the vector $x=\left[x_{1}, x_{2}, \ldots, x_{N}\right]^{T}$ where $N=L_{1} N_{1} \times L_{2} N_{2}$. Namely, $\mathbf{x}$ is the ideal un-degraded image that is sampled at orabove the Nyquist rate from a continuous scene which isassumed to be bandlimited. Now, let the parameters $L 1$ and $L 2$ represent the down-sampling factors in the observationmodel for the horizontal and vertical directions, respectively.Thus, each observed LR image is of size $L_{1} N_{1} \times L_{2} N_{2}$. Let the $k$ th LR image be denoted in lexicographicnotation as $y_{k}=\left[y_{k, 1}, y_{k, 2}, \ldots, y_{k, M}\right]^{T}$ for $k=1,2, \ldots, p$ and $M=L_{1} N_{1} \times L_{2} N_{2}$. Now, it is assumed that xremains constant during the acquisition of the multipleLR images, except for any motion and degradation allowedby the model. Therefore, the observed LR imagesresult from warping, blurring, and subsampling operatorsperformed on the HR image $\mathbf{x}$. Assuming that eachLRimage is corrupted by additive noise, we can then represent the observation model as [3],

$$
y_{k}=D B_{k} M_{k} X+n_{k} \text { for } 1 \leq k \leq p
$$

Where $M_{k}$ is awarpmatrix of size $L_{1} N_{1} L_{2} N_{2} \times L_{1} N_{1} L_{2} N_{2}, B_{k}$ represents a $L_{1} N_{1} L_{2} N_{2} \times L_{1} N_{1} L_{2} N_{2}$ blur matrix, $D$ is a $\left(N_{1} N_{2}\right)^{2} \times L_{1} N_{1} L_{2} N_{2}$ subsampling matrix, and $n_{k}$ represents a lexicographically ordered noise vector. Ablock diagram for the observation model is illustrated in Fig.2.

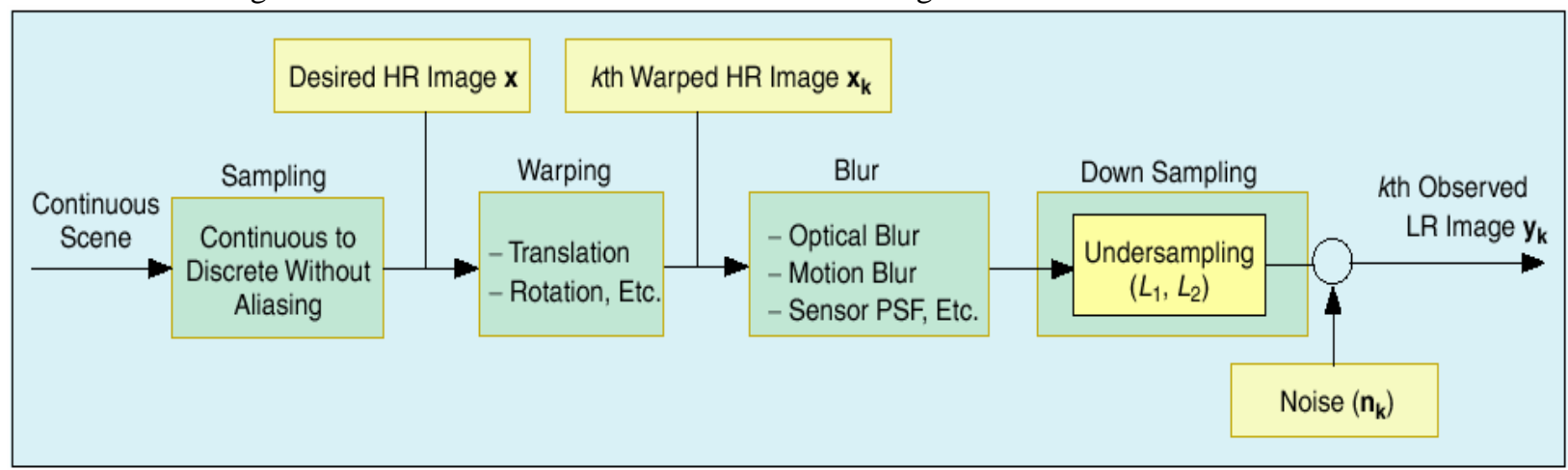

Fig.2 Observation Model 


\section{Super-resolution techniques}

Image super resolution techniques can be mainly categorized as reconstruction based techniques and learning based techniques.

Table 1 Comparison between Frequency Domain and Spatial Domain Super Resolution Techniques

\begin{tabular}{|l|l|l|}
\hline & Frequency Domain & Spatial Domain \\
\hline Observation model & Frequency domain & Spatial domain \\
\hline Motion Model & Global Translation & Almost Unlimited \\
\hline Degradation model & Global translation & Almost unlimited \\
\hline Noise model & Limited & Almost unlimited \\
\hline A-priori information & Limited & Almost unlimited \\
\hline Simplicity & Very Simple & Generally Complex \\
\hline Computational Cost & Low & High \\
\hline Regularization & Limited & Excellent \\
\hline Extensibility & Poor & Excellent \\
\hline Applicability & Limited & Wide \\
\hline Performance & Good for Specific Application & Good \\
\hline
\end{tabular}

In learning based approach, the relationship between an LR image and its corresponding high resolution (HR) image is examined via a pair of LR and HR patches. The training data is used to predict the higher-resolution image[4]. Reconstruction based approach can be employed in the frequency domain or spatial domain. Reconstruction based approach require either single image or multiple low resolution image. Simplicity in theory is a major advantage of the frequency domain approach. In addition, the frequency approach is also convenient for parallel implementation. However, this approach allows low flexibility to add priori constraints, noise models, and spatially varying degradation models. Thus, the development in practical use is limited. On the other hand, spatial domain techniques are more flexible in incorporating priori constraints and have better performance in reconstructed images. Nevertheless, these methods also have drawbacks such as complicated theoretical work and relatively large amount of computation load. A comparison of the two main classes of super-resolution techniques, frequency domain and spatial domain, is found in Table 1[5-9].

\subsection{Frequency Domain Approach}

The super-resolution problem was posed, along with a frequency domain solution, by Tsai and Huang[10]. Prior to their paper, interpolation was the best technique for increasing the resolution of images. The frequency domain approach makes explicit use of the aliasing that exists in each LR image to reconstruct an HR image. Tsai and Huang [10] first derived a system equation that describes the relationship between LR images and a desired HR image by using the relative motion between LR images. The frequency domain approach is based on the following three principles: i) the shifting property of the Fourier transform, ii) the aliasing relationship between the continuous Fourier transform (CFT) of an original HR image and the discrete Fourier transform (DFT) of observed LR images, iii) and the assumption that an original HR image is band limited. These properties make it possible to formulate the system equation relating the aliased DFT coefficients of the observed LR images to a sample of the CFT of an unknown image. For example, let us assume that there are two 1-D LR signals sampled below the Nyquist sampling rate. From the above three principles, the aliased LR signals can be decomposed into the de-aliased HR signal as shown in Fig. 3.

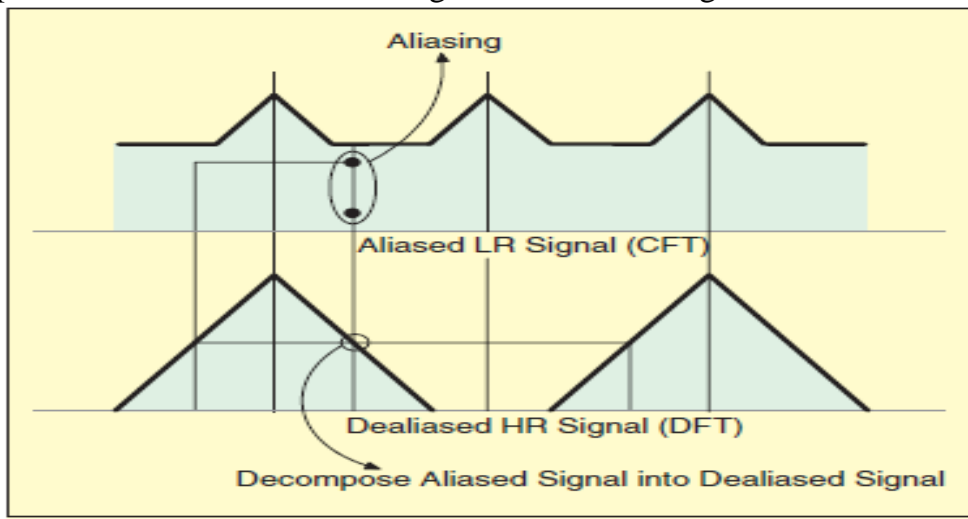

Fig.3. Aliasing relationship between LR image and HR image 
Frequency-domain super resolution method typically rely on familiar Fourier transform properties, specifically the shifting and sampling theorems. Since these properties are generally very well known and understood, the frequency domain approaches are easy to grasp and are intuitively appealing. Many of the frequency-domain approaches are based on assumptions which enable the use of efficient procedures for computing the restoration, the most important of which is the Fast Fourier Transform (FFT).

Let $x\left(t_{1}, t_{2}\right)$ denote a continuous HR image and $X\left(w_{1}, w_{2}\right)$ be its CFT. The global translations, which are the only motion considered in the frequency domain approach, yield the $k^{\text {th }}$ shifted image of $x_{k}\left(t_{1}, t_{2}\right)=x\left(t_{1}+\delta_{k 1}, t_{2}+\delta_{k 2}\right)$, where $\delta_{k 1}$ and $\delta_{k 2}$ are arbitrary but known values, and $k=1,2, . ., p$. By the shifting property of the CFT, the CFT of the shifted image, $X_{k}\left(w_{1}, w_{2}\right)$, can be written as

$$
X_{k}\left(w_{1}, w_{2}\right)=\exp \left[j 2 \pi\left(\delta_{k 1} w_{1}+\delta_{k 2} w_{2}\right)\right] X\left(w_{1}, w_{2}\right)
$$

The shifted image $x_{k}\left(t_{1}, t_{2}\right)$ is sampled with the sampling period $T 1$ and $T 2$ to generate the observed LR image $y_{k}\left(n_{1}, n_{2}\right)$. Fromthe aliasing relationship and the assumption of bandlimitedness of $X\left(w_{1}, w_{2}\right)$ $\left(\left|X\left(w_{1}, w_{2}\right)\right|\right)=0$ for $\left.\left|w_{1}\right| \geq\left(L_{1} \pi / T_{1}\right),\left|w_{2}\right| \geq\left(L_{2} \pi / T_{2}\right)\right)$, the relationship between the CFT of theHRimage and the DFT of the $k^{\text {th }}$ observed LR image can be written as [11]

$$
\gamma_{k}\left(\Omega_{1}, \Omega_{2}\right)=\frac{1}{T_{1} T_{2}} \sum_{n_{1}=0}^{L_{1}-1} \sum_{n_{2}=0}^{L_{2}-1} X_{k} \times\left[\frac{2 \pi}{T_{1}}\left[\frac{\Omega_{1}}{N_{1}}+n_{1}\right], \frac{2 \pi}{T_{2}}\left[\frac{\Omega_{2}}{N_{2}}+n_{2}\right]\right]
$$

By using lexicographic ordering for the indices $n 1, n 2$ on the right-hand side and $k$ on the left-hand side, a matrix

vector form is obtained as:

$$
Y=\Phi X
$$

whereY is a $p \times 1$ column vector with the $k^{\text {th }}$ element of the DFT coefficients of $y_{k}\left[n_{1}, n_{2}\right], \mathbf{X}$ is a $L_{1} L_{2} \times 1$ column vector with the samples of the unknown CFT of $x\left(t_{1}, t_{2}\right)$, and $\Phi$ is a $p \times L_{1} L_{2}$ matrix which relates the DFT of the observed LR images to samples of the continuous HR image. Therefore, the reconstruction of a desired HR image requires us to determine $\Phi$ and solve this inverse problem. An extension of this approach for a blurred and noisy image was provided by Kim et al. [12], resulting in a weighted least squares formulation. In their approach, it is assumed that all LR images have the same blur and the same noise characteristics. This method was further refined by Kim and Su [13] to consider different blurs for each LR image. Here, the Tikhonov regularization method is adopted to overcome the ill-posed problem resulting from blur operator. Bose et al. [14] proposed the recursive total least squares method for SR reconstruction to reduce effects of registration errors (errors in $\Phi$ ). A discrete cosine transform (DCT)-based method was proposed by Rhee and Kang [15]. They reduce memory requirements and computational costs by using DCT instead of DFT. They also apply multichannel adaptive regularization parameters to overcome ill-posedness such as underdetermined cases or insufficient motion information cases. Theoretical simplicity is a major advantage of the frequency domain approach. That is, the relationship between LR images and the HR image is clearly demonstrated in the frequency domain. The frequencymethod is also convenient for parallel implementation capable of reducing hardware complexity.

\subsection{Spatial Domain Approach}

In this class of SR reconstruction methods, the observation model is formulated, and reconstruction is effected in the spatial domain. The linear spatial domain observation model can accommodate global and nonglobal motion, optical blur, motion blur, spatially varying PSF, non-ideal sampling, compression artifacts and more. Spatial domainreconstruction allows natural inclusion of (possibly nonlinear) spatial domain a-priori constraints (e.g. Markov random fields or convex sets) which result in bandwidth extrapolation in reconstruction. Consider estimating a SR image $\mathrm{z}$ from multiple LR images $y_{r}, r \in\{1,2, \ldots, R\}$. Images are written as lexicographically ordered vectors. $y_{r}$ and $\mathrm{z}$ are related as $y_{r}=H_{r} z$. The matrix $H_{r}$, which must be estimated, incorporates motion compensation, degradation effects and subsampling. The observation equation may be generalized to $\mathrm{Y}=\mathrm{Hz}+\mathrm{N}$ where $Y=\left[y_{1}{ }^{T} \ldots y_{R}{ }^{T}\right]^{T}$ and $H=\left[H_{1}{ }^{T} \ldots H_{R}{ }^{T}\right]^{T}$ with $\mathrm{N}$ representing observation noise. 
Since the superresolution problem is fundamentally ill-posed, incorporation of prior knowledge is essential to achieve good results. A variety of techniques exist for the super-resolution problem in the spatial domain. These solutions include interpolation, deterministic regularized techniques, stochastic methods, iterative back projection, and projection onto convex sets among others. The primary advantages to working in the spatial domain are support for unconstrained motion between frames and ease of incorporating prior knowledge into the solution.

\subsubsection{Interpolation of Non Uniformly Spaced Samples}

Registering a set of LR images using motion compensation results in a single, dense composite image of non uniformly spaced samples. A SR image may be reconstructed from this composite using techniques for reconstruction from non-uniformly spaced samples. Unfortunately, this technique generally works very poorly because of some inherent assumptions; the main problem being that camera sensors do not act as impulse functions. Since the observed data result from severely under sampled, spatially averaged areas, the reconstruction step (which typically assumes impulse sampling) is incapable of reconstructing significantly more frequency content than is present in a single LR frame. Degradation models are limited, and no a-priori constraints are used. There is also question as to the optimality of separate merging and restoration steps.

\subsubsection{Deterministic Regularization}

The deterministic regularized SR approach solves the inverse problem by using the prior information about the solution which can be used to make the problem well posed. For example, CLS can be formulated by choosing an $\mathbf{x}$ to minimize the Lagrangian [16]

$$
\left[\sum_{k=1}^{p}\left\|y_{k}-W_{k} x\right\|^{2}+\alpha\|C X\|^{2}\right]
$$

where the operator $\mathbf{C}$ is generally a high-pass filter, and $\|$.$\| represents a l 2$-norm. In eq(5), a priori knowledge concerning a desirable solution is represented by a smoothness constraint, suggesting that most images are naturally

smooth with limited high-frequency activity, and therefore it is appropriate to minimize the amount of high-pass energy in the restored image. In eq(5), $\alpha$ represents the Lagrange multiplier, commonly referred to as the regularization parameter, that controls the tradeoff between fidelity to the data (as expressed by $\sum_{k=1}^{p}\left\|y_{k}-W_{k} x\right\|^{2}$ ) andsmoothness of the solution (as expressed by $\|C X\|^{2}$ ). TheLarger values of $\alpha$ will generally lead to a smoothersolution. This is useful when only a small number of LR imagesare available (the problem is underdetermined) orthe fidelity of the observed data is low due to registrationerror and noise. On the other hand, if a large number ofLR images are available and the amount of noise is small,smallawill lead to a good solution. The cost functional ineq(6) is convex and differentiable with the use of a quadraticregularization

term. Therefore, we can find a unique estimateimage $x$ which minimizes the cost functional in eq(5).

One of the most basic deterministic iterative techniquesconsiders solving

$$
\left[\sum_{k=1}^{p} W_{k}^{T} W_{k}+\alpha C^{T} C\right] \hat{X}=\sum_{k=1}^{p} W_{k}^{T} y_{k}
$$

and this leads to the following iteration for $x$ :

$$
\hat{X}^{(n+1)}=\hat{X}^{(n)}+\beta\left[\sum_{k=1}^{p} W_{k}^{T}\left(y_{k}-W_{k} \hat{X}^{(n)}\right)-\alpha C^{T} C \hat{X}^{(n)}\right]
$$

where $\beta$ represents the convergence parameter and $W_{k}^{T}$ contains an up-sampling operator and a type of blur andwarping operator.

\subsubsection{Projection Onto Convex Sets(POCS)}

Another method for reducing the space of possible reconstructions is projection onto convex sets (POCS). The POCS method describes an alternative iterative approach to incorporating prior knowledge about the solution into the reconstruction process. With the estimates of registration parameters, this algorithm simultaneously solves the restoration and interpolation problem to estimate the SR image. This is a set-theoretic approach where each piece of a priori knowledge is formulated as a constraining convex set. Once the group of 
convex sets is formed, an iterative algorithm is employedto recover a point on the intersection of the convex sets,

$$
g_{i+1}=P_{M} P_{M-1} \ldots . . P_{2} P_{1}\left\{g_{i}\right\}
$$

where $P_{j}$ is the projection of a given point onto the $j^{\text {th }}$ convex set and $M$ is the number of convex sets. In essence, we are restricting the final restored image to lie on the intersection of the constrainingsets, $\left\{P_{j}\right\}_{j=1}^{M}$. The reason we require convex sets is that convergenceis guaranteed for the case where each set is convex.

One potential group of convex sets is based on the $l 2$ distance measure,

$$
G_{k}=\left\{g\left\|W_{k} g-y_{k}\right\|^{2} \leq 1\right\}, 1 \leq k \leq K
$$

This defines a set of ellipsoids (one for each input image) and restrictsthe final solution to lie inside the ellipsoids. Other possibleconvex sets include ones based on the $¥$ norm, those imposingsmoothness, and those constraining the image intensity to be positive. Two problems with the POCS approach are that uniqueness isnot guaranteed for the final recovered image and that defining theprojections $P_{j}$ can be difficult.

\subsubsection{Iterative Back Projection(IBP)}

Super resolution of the image is model as an inverse problem. That is the goal of super resolution is to reverse the effect of the down sampling, blurring and warping that relate the LR image to desired HR image. Mathematically it

is written as

$$
y=(X * W) \downarrow S
$$

Where, $X$ is original HR image; $y$ is LR image; $W$ is degeneration function; $\downarrow s$ is down sampling process.

In IBP approach HR image is estimated by back projecting the difference between the simulated LR image and captured LR on interpolated image. This iterative process of SR does iterations until the minimization of the cost function is achieved. Block diagram of IBP algorithm is given in Fig. 4. Mathematically the SR step according to IBP is written as

$$
X=X^{(0)}+X_{e}
$$

Where, $\mathrm{X}$ is interpolated image; $X_{e}$ is error correction.

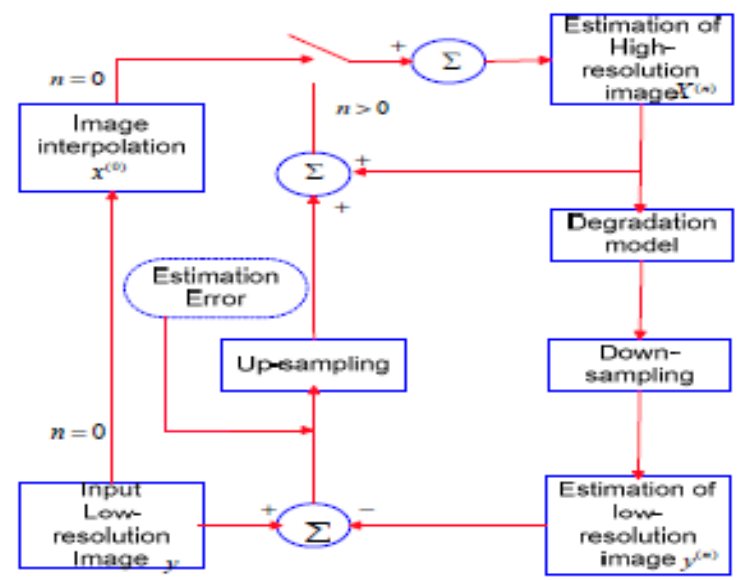

Fig. 4 Simple IBP Algorithm[17]

Given only one LR input image, the updating procedure can be summarized as doing the following two steps iteratively:

1) Compute the LR error as

$$
X_{e}^{(n)}=\left(y-y^{(n)}\right) \uparrow S
$$

Estimation of the simulated LR image is given as

$$
y^{(n)}=\left(X^{(n)} * W\right) \downarrow S
$$

2) Update the HR image by back-projecting the error as equation (11).

\subsubsection{Proposed IBP using Canny Edge Detection}


Though IBP method can minimize the restoration error significantly in iterative manner and gives good effect, it projected the error back without edge guidance. In proposed algorithm, extra high frequency information is added by Canny edge detection and difference error of up-sampled images from initial and simulated LR images and so that it works as edge preserving algorithm. Block diagram of proposed algorithm is shown in Fig. 5. Steps for proposedalgorithm are given in Table II.

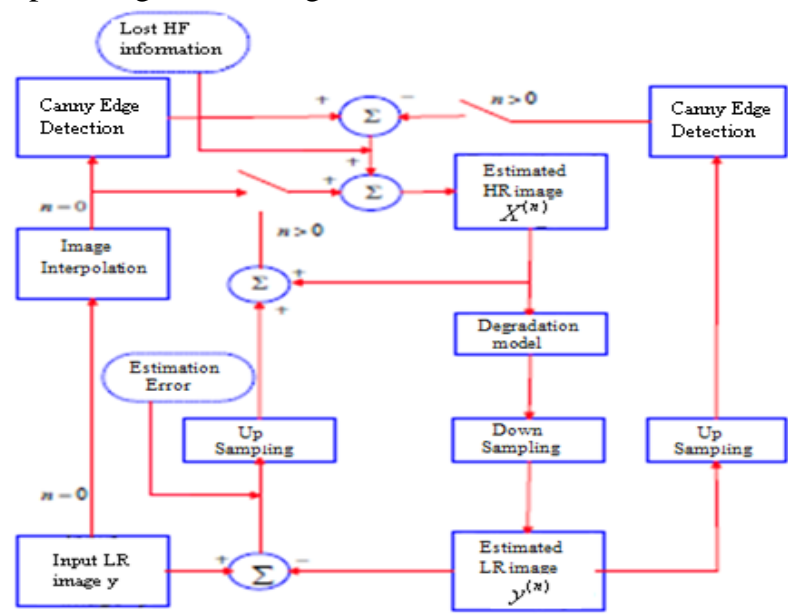

Fig. 5 Canny Edge Detection Algorithm

Mathematically SR according to proposed algorithm is written as

$$
X=X^{(0)}+X_{e}+X_{H}
$$

Where, $X^{(0)}$ is initial interpolated image; $X_{e}$ is error correction; $X_{H}$ is high frequency estimation given by

$$
X_{H}=\left(X * H_{\text {Canny }}\right)
$$

Where $X_{H}$ is the estimated HR image, $H_{\text {Canny }}$ is the Canny Edge High Pass filter.

In Summary, proposed algorithm in mathematical form is expressed as below. The estimated HR image after $n$ iterations is given by

$$
X^{(n+1)}=X^{(n)}+X_{e}+X_{H}^{(n)}
$$

The estimation of the high frequency is given as

$$
X_{H}^{(n)}=X_{H}^{(0)}-\left\{\left(\left(y^{(n)}\right) \uparrow S\right) * H_{\text {Canny }}\right\}
$$

Where $X_{H}^{(0)}$ is high frequency component of image $X^{(0)}$. The formula for $X_{H}^{(0)}$ is given as

$$
X_{H}^{(0)}=\left(X^{(0)} * H_{\text {Canny }}\right)
$$

So, the final iterations process given in (13) is rewritten for the combination of IBP and Canny Edge as

$$
X^{(n+1)}=X^{(n)}+\left(y-y^{(n)}\right) \uparrow S+\left\{X_{H}^{(0)}-\left\{\left(\left(y^{(n)} \uparrow S\right) * H_{\text {Canny }}\right\}\right\}\right.
$$

Table II Steps of Canny Edge Detection Algorithm

\begin{tabular}{ll} 
NO & Read ground truth image \\
2 & Apply Gaussian blurring and down sampling to generate observed LR image \\
3 & Apply initial interpolation on observed LR image for initial estimation of HR image \\
4 & Apply Canny Edge on initial estimated HR image to obtain high frequency component \\
5 & Apply degradation on initial estimated HR image to generate simulated LR image \\
6 & Apply Canny Edge on simulated LR image \\
7 & Subtract simulated LR from Observed LR image that gives error correction \\
8 & Subtract step 6 output from step 4 output to get lost high frequency component \\
9 & Take summation of step 3 output with step 8 output. This will give improvement in quality of initial \\
& $\begin{array}{l}\text { estimated HR image } \\
10\end{array}$ \\
11 & Now, this improved HR becomes our initial estimated HR image for the second iteration \\
\hline
\end{tabular}




\section{Result and Discussion}

To evaluate the SR systems effectively, this work assumes that the original HR images exist and the image qualitydegradation is resulted from Gaussian blurring, and such Gaussian blurring function is known.

To present the performance of this algorithms, several images are tested and results are compared with Nearest Neighbourhood interpolation(NN), Bilinear interpolation (BI), bicubic interpolation (BC), POCS, IBP and IBP + Canny Edge Detection. The image quality is determined based on PSNR evaluation.

As performance criteria, Peak Signal to Noise Ratio (PSNR) is calculated. The mathematical equations for $M \times N$ image analysis are as given bellow[18].

$$
\begin{aligned}
& M S E=\frac{\sum_{i} \sum_{j}\left[X(i, j)-X^{(n)}(i, j)\right]^{2}}{M \times N} \\
& P S N R=10 \log _{10}\left(\frac{255 \times 255}{M S E}\right)
\end{aligned}
$$

Where, $X(i, j)$ is the original HR image and $X^{(n)}(i, j)$ is the estimated HR image through this algorithm. The image quality is determined based on PSNR evaluation. A good reconstruction algorithm generally provides low value of MSE and high value of MSSIM and PSNR. The resultant images are shown in figure 6 and 7 . PSNR is obtained using various techniques are given in Table III.

Table III PSNR Comparison

\begin{tabular}{|l|l|l|l|l|l|l|}
\hline \multirow{3}{*}{$\begin{array}{c}\text { Test } \\
\text { Image }\end{array}$} & \multicolumn{7}{c|}{ Single Frame Algorithms } \\
\cline { 2 - 7 } & NN & BI & Bicubic & POCS & IBP & $\begin{array}{l}\text { IBP } \\
\text { Canny } \\
\text { Edge }\end{array}$ \\
\hline Apple & 26.6500 & 29.1617 & 31.6337 & 30.7417 & 28.7719 & 42.3146 \\
\hline Football & 26.9158 & 29.3864 & 30.4000 & 30.3136 & 29.2219 & 42.8838 \\
\hline Greens & 19.9802 & 22.1634 & 24.6540 & 28.2204 & 22.1575 & 34.4326 \\
\hline Monkey & 29.6891 & 31.2404 & 33.4968 & 32.7840 & 31.0809 & 43.6170 \\
\hline Flower & 29.1997 & 32.0320 & 33.9559 & 32.4331 & 31.7295 & 46.0387 \\
\hline Fruits & 28.4520 & 31.0726 & 34.1511 & 32.9744 & 30.9810 & 43.7963 \\
\hline
\end{tabular}

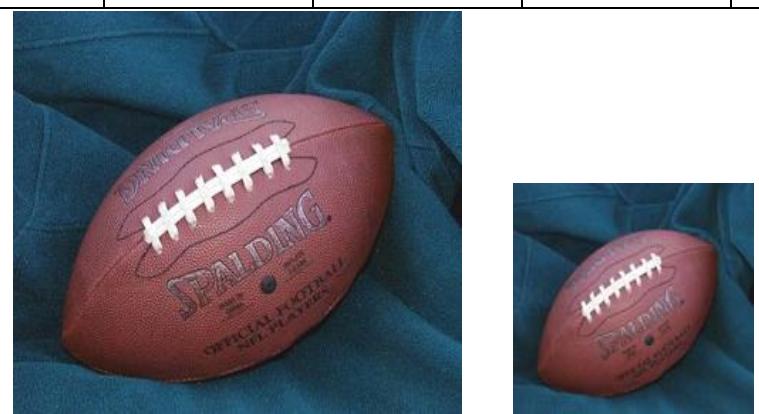
(a) Original Image
(b) LR Image

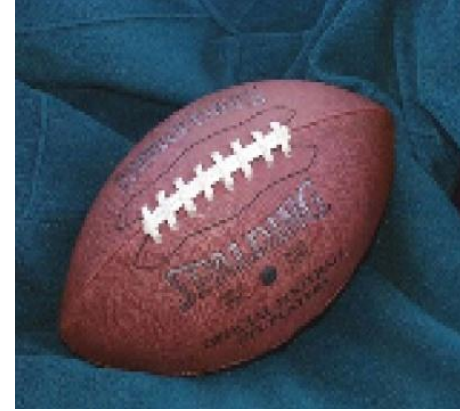

(c) NN Interpolation

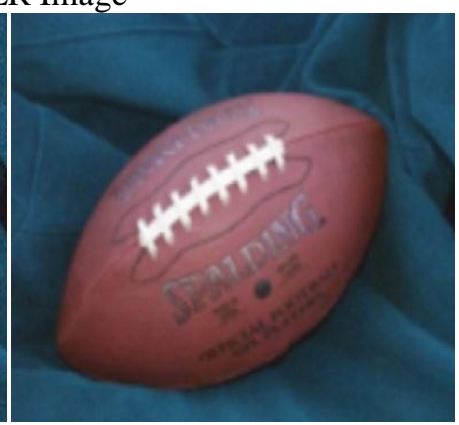

(d) Bilinear Interpolation 


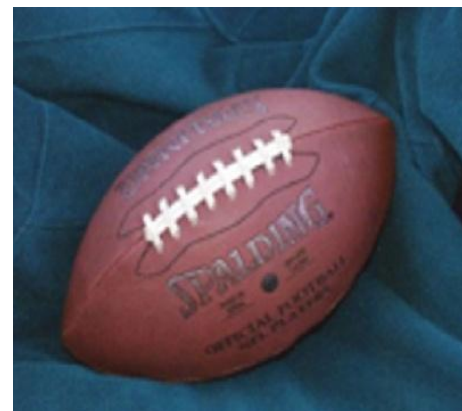

(e) Bicubic Interpolation

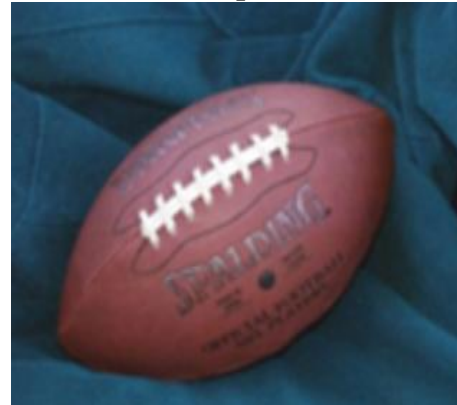

(g) IBP

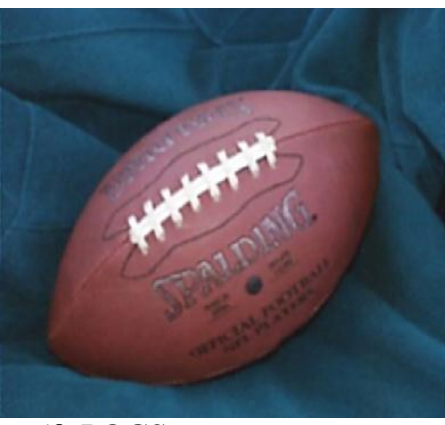

(f) POCS

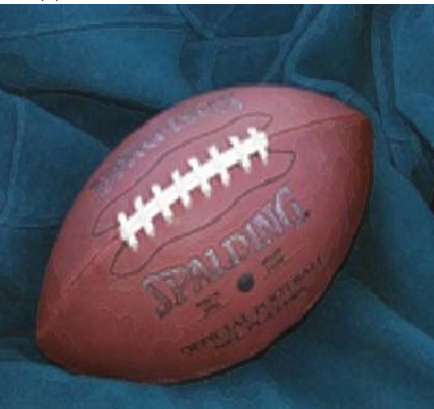

(h) IBP + Canny Edge

Fig.6. Resultant football Image using different Algorithms

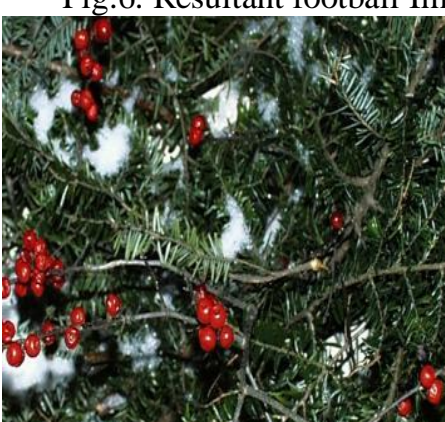

(a)Original Image

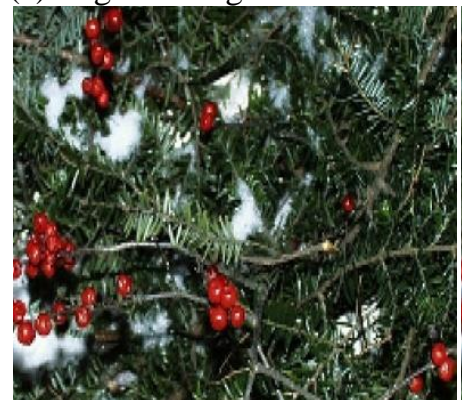

(c) NN Interpolation (d) Bilinear Interpolation

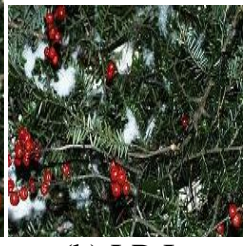

(b) LR Image
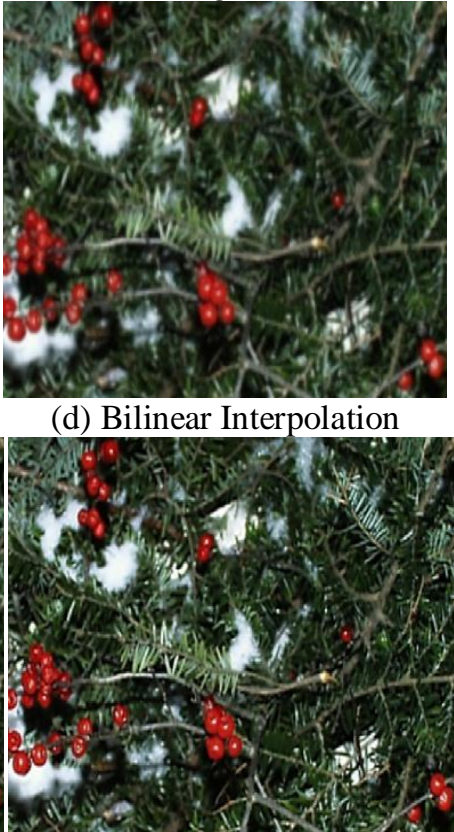

(f) POCS

(e) Bicubic Interpolation 


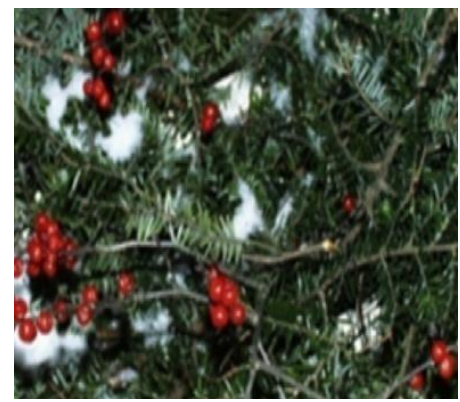

(g) IBP

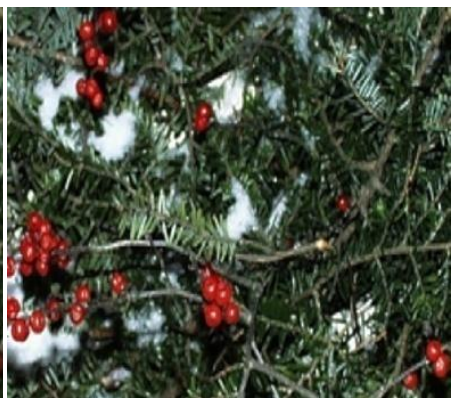

(h) IBP + Canny Edge

Fig.7. Resultant Greens Image using different Algorithms

\section{Future Research Directions}

we have identified some new directions for future research. The first asks the question "Can we design camera sensors that are optimal for super-resolution algorithms?".Current CCDs act like box functions, spatially integrating over a uniform rectangular region for each pixel. It is likely that other types of sampling, such as integration over a Gaussian function, would yield better results.

Another direction is to combine super-resolution with dense stereo reconstruction. Dense stereo reconstruction is the process of estimating the depth of every point in the scene from two or more input images taken from different poses. Current techniques typically estimate depths for every pixel in one of the images, but it should be possible to obtain depths on a finer grid by combining super-resolution techniques with dense stereo.

A third potential problem is to combine high dynamic range imaging with super-resolution. High dynamic range imaging involves taking multiple images from the same viewpoint at different exposures and then combining the images to obtain an image of higher dynamic range. By taking multiple images at different exposures and with slight translational offsets it might be possible to obtain a super-resolved high dynamic range image more efficiently than if the two stages are done independently.

\section{Conclusion}

The SR imaging has been one of the fundamental image processing research areas. It can overcome or compensate the inherent hardware limitations of the imaging system to provide amore clear image with a richer and informative content. We have provided a review of the current state of super-resolution research, covering the past, present, and future of the problem. A standard image formation model was first introduced, followed by summaries of the major classes of algorithms including frequency domain approaches, deterministic regularization, projection onto convex sets, Iterative Back Projection and Proposed IBP with Canny Edge Detection. In this survey paper, our goal is to offer new perspectives and outlooks of SR imaging research, besides giving an updated overview of existing SR algorithms. It is our hope that this work could inspire more image processing researchers endeavoring on this fascinating topic and developing more novel SR techniques along the way. Finally we discussed potential future directions for research.

\section{References}

[1] Assaf Zomet, Alex Rav-Acha, Shmuel Peleg, "Robust Super-Resolution" ,ISBN 0-7695-1272-0/01 \$10.00 (C) 2001 IEEE.

[2] D. Capel and A. Zisserman. Super-resolution enhancement of text image sequences. In Int. Conf. Pattern Recognition, pages Vol I: $600.605,2000$

[3] Sung Cheol Park, Min Kyu Park,and Moon Gi Kang“Super Resolution:A Technical Overview,"IEEE SIGNAL PROCESSING MAGAZINE 1053-5888/03/\$17.00@2003IEEE.

[4] C. V. Jiji and Subhasis Chaudhuri, 2006, "Single-Frame Image Super-resolution through Contourlet Learning", EURASIP Journal on Applied Signal Processing, VOL.2006, Article ID 73767, pp 1-11.

[5] David Capel and Andrew Zisserman, 2000, "Super-resolution Enhancement of Text Image Sequences", IEEE Comput. Soc, Proceedings 15th International Conference on Pattern Recognition ICPR, VOL. 1, No. 1, pp-600-605.

[6] Sina Farsiu, M. Dirk Robinson, Michael Elad, and Peyman Milanfar, 2004, "Fast and Robust Multiframe Super Resolution", IEEE transactions on image processing, VOL. 13, NO. 10, pp 1327-1344.

[7] Xueting Liu, Daojin Song, Chuandai Dong and Hongkui Li, 2008, "MAP-Based Image Super-resolution Reconstruction", World Academy of Science, Engineering and Technology 37, pp 208-211.

[8] Kamal Nasrollahi, “ A Computer Vision Story On Video Sequences: From Face Detection to Face Super Resolution using Face Quality Assessment”, Faculty of engineering and science, IEEE Member, AALBORG UNIVERSITY 2011.

[9] BORMAN, S., AND STEVENSON, R. 1998. Super-resolution from image sequences - A review. In Proceedings of the 1998 Midwest Symposium on Circuits and Systems, IEEE, Notre Dame, IN, USA, 374-378.

[10] TSAI, R. Y., AND HUANG, T. S. 1984. Multi-frame image restoration and registration. In in Advances in Computer Vision and Image Processing, 317-339.

[11] A.M. Tekalp, Digital Video Processing. Englewood Cliffs, NJ: Prentice Hall, 1995.

[12] S.P. Kim, N.K. Bose, and H.M. Valenzuela, "Recursive reconstruction of high resolution image from noisy under sampled multi frames," IEEE Trans. Acoust., Speech, Signal Processing, vol. 38, pp. 1013-1027, June 1990. 
[13] S.P. Kim and W.Y. Su, "Recursive high-resolution reconstruction of blurred multiframe images," IEEE Trans. Image Processing, vol. 2, pp. 534-539, Oct. 1993.

[14] N.K. Bose, H.C. Kim, and H.M. Valenzuela, "Recursive implementation of total least squares algorithm for image reconstruction from noisy, under sampled multi frames," in Proc. IEEE Conf. Acoustics, Speech and Signal Processing, Minneapolis, MN, Apr. 1993, vol. 5, pp. 269-272.

[15] S.H. Rhee and M.G. Kang, "Discrete cosine transform based regularized high-resolution image reconstruction algorithm," Opt. Eng., vol. 38, no. 8, pp. 1348-1356, Aug. 1999.

[16] A.K. Katsaggelos, Ed. Digital Image Restoration. Heidelberg, Germany: Springer-Verlag. Springer. vol. $23,1991$.

[17] V. B. Patel, Chintan K. Modi, C. N. Paunwala and S. Patnaik, "Hybrid Approach for Single Image Super Resolution using ISEF and IBP”, in IEEE Conference on Communication System and Network Technology (CSNT), 03-05 june, 2011.

[18] Liyakathunisa and C.N .Ravi Kumar," A NOVEL SUPER RESOLUTION RECONSTRUCTION OF LOW REOSLUTION IMAGESPROGRESSIVELY USING DCT AND ZONAL FILTER BASED DENOISING”, International Journal of Computer Science \& Information Technology (IJCSIT), Vol 3, No 1, Feb 2011. 